and pathogenic Actinomyces species in man and described serological methods developed for species and strain identification. Studies on gastroenteritis in breast fed infants by Dr A. T. Willis, Catherine L. Bullen and Kathleen Williams (Luton and Dunstable Hospital, Luton) illustrated the importance of Bifidobacteria in the gut at an early age. A rapid method for identifying Bifidobacteria species and therefore analysing different gut populations was described by Drs M. Catteau and $\mathrm{F}$. Poncelet and Professor $\mathrm{M}$. Beerens (CERTIA, Lille).

\section{PLANT BIOCHEMISTRY Nitrogen Metabolism}

from a Correspondent THE IUB/IUBS symposium on nitrogen metabolism in plants, held at the University of Leeds from July 10 to 14 , showed the great progress that has been made recently in understanding the processes of nucleic acid and protein synthesis in plants.

Drs A. B. Legocki (College of Agriculture, Poznan) and J. E. Allende (University of Santiago) discussed poly. peptide chain elongation on plant $80 \mathrm{~S}$ ribosomes. They have isolated two elongation factors; EF1 is the factor responsible for promoting the binding of amino-acyl-tRNA to the ribosome and EF2, the translocation factor, is necessary for the movement of peptidyltRNA from the amino-acyl-tRNA site to the peptidyl-tRNA site on the ribosome. Both elongation factors require GTP for their action, and there is evidence that EF1 changes its configuration by loss of a subunit on binding with amino-acyl-tRNA. Dr A. Marcus (Institute for Cancer Research, Philadelphia) discussed the initiation of protein synthesis. Using tobacco mosaic virus RNA as a natural messenger in an in vitro system from wheat embryos, he has found that initiation proceeds as follows. The messenger RNA binds with the $40 S$ subunit of the ribosome; initiating methionyl-tRNA then binds to the peptidyl-tRNA site on the 40 S subunit and, finally, the initiation complex is completed by addition of the $60 \mathrm{~S}$ sub. unit of the ribosome. The process requires ATP, GTP and two initiation factors. It is clear from these studies that although the mechanism of protein synthesis in plants is similar to that in bacteria, there are nevertheless importand differences between the two synthesizing systems.

It is now accepted that, although plastids and mitochondria contain their own DNA and protein synthesizing systems, they are not completely autonomous. Nevertheless, as Drs R. J. Ellis and M. R. Hartley (University of Warwick) showed, it is possible to obtain isolated chloroplast preparations which will bring about light-dependent synthesis of protein and RNA. The bulk of the protein made by such a chloroplast preparation consists of the large subunit of fraction I protein, although several other proteins, including at least one membrane-bound protein, are also synthesized. The product of RNA synthesis in the isolated chloroplasts is a large precursor of chloroplast ribosomal RNA (molecular weight $\left.3.0 \times 10^{6}\right)$. It has not yet been possible to demonstrate in vitro the processing of this precursor to form the ribosomal RNA.

The interactions of nuclear and plastid genomes in controlling plastid development are very complex. Professor L. Bogorad (Harvard University) has isolated nine mutants of Chlamydomonas which exhibit changes or deficiencies in chloroplast ribosomal proteins. Eight of these mutations may be assigned to nuclear genes, whereas the ninth is inherited uniparentally, suggesting a location in the plastid genome. Professor R. Hagemann (University of Halle-Wittenberg) has studied a mutant of Pelargonium which lacks plastid ribosomal RNA. The mutation, which is located in the plastid genome, results in the formation of very small plastids which contain no chloro. phyll, internal membranes or ribosomes. The plastids, however, do have a complete double outer membrane, are able to replicate, and therefore presumably to synthesize DNA. Evidently the proteins necessary for these latter features of plastid growth are not made on plastid ribosomes.

The view that plant hormones act directly on the genome is not now generally held. Nevertheless, plant hormones are obviously involved in the regulation of nucleic acid and protein synthesis in vivo. Professor F. Skoog (University of Wisconsin) suggested possible modifications of tRNA caused by cytokinins, and Dr L. Dure (University of Georgia) showed that abscisic acid is involved in preventing the precocious germination of cotton seed embryos, and that this action is dependent on RNA synthesis. Before such effects are understood, it is necessary to carry out biophysical studies of the rapid short-term effects of plant hormones, as was suggested by $\mathrm{Dr} \mathbf{R}$. L. Rayle (California State University, San Diego).

\section{Viral Messengers}

THE messenger RNAs of eukaryctic cells have long half-lives so that it has been suggested that control of the rate of synthesis of particular proteins may occur at the level of translation rather than transcription. Finding suitable experimental systems to test this idea has, however, proved difficult, which is one reason why the work reported by Perlman, Hirsch and Penman in Nature New Biology next Wednesday (August 2) will be welcomed.

Late during the infection of $\mathrm{HeLa}$ cells by adenoviruses almost all proteins made are viral. In such cells the polysomes are much smaller than in uninfected HeLa cells even though adenovirus proteins are on average at least as large as cell proteins. One explanation of this discrepancy is that the rate of initiation of translation of the viral mRNAs is limiting and much slower than the rate of initiation of translation of cell messengers so that the viral messengers are less densely packed with ribosomes than cell polysomes.

This idea is supported by the fact that when the rate of chain elongation is slowed some five-fold, by adding small amounts of cycloheximide, the size of viral polysomes in infected cells increases. In these circumstances chain elongation rather than initiation is presumably limiting. Moreover, the finding that the ratio of the different adenovirus proteins made in the presence of cyclcheximide differs from the ratio of proteins made in its absence suggests that adenovirus messengers are translated at different rates. That is the conclusion which Perlman et al. draw from comparisons of the pattern of synthesis of adenovirus proteins, in the presence and absence of cycloheximide, in HeLa cells cultured at $37^{\circ} \mathrm{C}$ and $40^{\circ} \mathrm{C}$. And because initiation of translation in HeLa cells is inhibited at elevated temperatures it seems likely that any differences in the rate of translation of adenovirus messengers in these cells stem from differences in the rate of chain initiation.

Many viral messengers, like their cellular counterparts, terminate in a $3^{\prime}$ sequence of some 50-200 adenylic acid residues. Whatever the role in vivo of these poly(A) tails they provide a convenient handle for fishing out messengers, and, as Zassenhaus and Kates report in the same issue of Nature New Biology, they can be exploited to achieve the efficient synthesis of complementary DNA by reverse transcriptase. The trick, of course, is to add a short oligomeric GT primer. This primer hydrogen bonds to the $3^{\prime}$ poly(A) sequence of the messenger RNA template and provides reverse transcriptase with a free 3 hydroxy group on which to initiate synthesis of complementary DNA. Zassenhaus and Kates chose vaccinia virus mRNA made in vitro as a model system but their findings should be of general application. 